\title{
Avaliação das atividades cicatrizante, anti-inflamatória tópica e antioxidante do extrato etanólico da Sideroxylon obtusifolium (quixabeira)
}

LEITE, N.S.'; LIMA, A.P.2; ARAÚJO-NETO, V. ${ }^{1 ;}$ ESTEVAM, C.S.'; PANTALEÃO, S.M. ${ }^{2}$; CAMARGO, E.A. ${ }^{1 ;}$ FERNANDES, R.P.M. ${ }^{\prime}$; COSTA, S.K.P. ${ }^{3}$; MUSCARÁ, M.N. ${ }^{3 ;}$ THOMAZZI, S.M. ${ }^{*}$

${ }^{1}$ Departamento de Fisiologia e ${ }^{2}$ Departamento de Biologia, Centro de Ciências Biológicas e da Saúde, Universidade Federal de Sergipe (UFS), Av. Marechal Rondon, S/N, Jardim Rosa Elze, São Cristóvão-SE, Brasil, CEP 49100-000. ' ${ }^{3}$ epartamento de Farmacologia, Instituto de Ciências Biomédicas, Universidade de São Paulo (USP), Av. Prof. Lineu Prestes, 1524, São Paulo-SP, Brasil, CEP 05508-900. *Autor para correspondência: sthomazzi@gmail.com

RESUMO: A planta Sideroxylon obtusifolium, conhecida popularmente como "quixabeira", é utilizada na medicina popular para o tratamento de processos inflamatórios e dolorosos. 0 objetivo deste trabalho foi avaliar a possível ação cicatrizante, bem como anti-inflamatória tópica, do extrato etanólico (EE) da entrecasca da S. obtusifolium no modelo de ferida e cicatrização cutânea. Para isso, ratos Wistar foram anestesiados, submetidos à incisão dorsal, e discos de esponja de álcool polivinílico foram inseridos subcutâneamente. Quarenta e oito horas após os animais foram eutanasiados avaliando-se medidas de tensão de ruptura nas cicatrizes da pele dorsal, a atividade de mieloperoxidase (MPO), e a contagem de células nos exsudatos das esponjas. A atividade sequestradora do radical 2,2-difenil-1-picrilhidrazil (DPPH) foi determinada para avaliar a capacidade antioxidante do EE. Observamos que a administração tópica, 2 vezes ao dia, do EE da S. obtusifolium (3-30 mg 0,4 mL-1 de creme Lanette ${ }^{\circledR}$ ) não alterou a tensão de ruptura das cicatrizes. Entretanto, o EE da S. obtusifolium (30 mg 0,4 mL-1 de creme Lanette ${ }^{\circledast}$ ) foi capaz de diminuir a atividade de MPO $(p<0,01)$ e a contagem celular $(p<0,05)$ no exsudato das esponjas, após $48 \mathrm{~h}$ da indução da ferida. O EE mostrou-se ativo como sequestrador do radical DPPH. Os valores de $\mathrm{IC}_{50}$ para o $\mathrm{EE}$ e ácido gálico foram semelhantes $(1,04 \pm 0,27 \mathrm{e}$ $1,15 \pm 0,02 \mu \mathrm{g} \mathrm{mL}^{-1}$ de DPPH, respectivamente). Concluímos que o EE da entrecasca da $S$. obtusifolium possui atividade anti-inflamatória através da administração tópica no modelo de cicatrização cutânea em ratos, além de seu potencial antioxidante.

Palavras-chave: Cicatrização, inflamação, mieloperoxidase, migração de leucócitos, Sapotaceae, Sideroxylon.

ABSTRACT: Evaluation of the cicatrizing, topical anti-inflammatory and antioxidant activities of the ethanol extract of Sideroxylon obtusifolium. The Sideroxylon obtusifolium is a medicinal plant known as "quixabeira" in Portuguese that has been used in folk medicine for the treatment of inflammatory and painful processes. The aim of this study was to evaluate the effect of the ethanol extract (EE) of $S$. obtusifolium on the cicatrization and local anti-inflammatory activities using a model of wound healing in rats. Wistar rats were submitted to a dorsal skin incision and polyvinyl alcohol sponge discs were implanted. Animals were euthanized after $48 \mathrm{~h}$, and the tensile strength measurement at the wound site, the myeloperoxidase (MPO) activity and the leukocyte counts in the wound exudates were evaluated. The 2,2-diphenyl-1-picrylhydrazyl (DPPH) radical scavenging activity was determined to evaluate the antioxidant capacity of the EE. We observed that the topical administration of the EE of $S$. obtusifolium (3-30 mg $0.4 \mathrm{~mL}^{-1}$ of Lanette ${ }^{\oplus}$ cream) twice a day did not alter the tensile strength, but it decreased the MPO activity $(p<0.01)$ and leukocyte infiltration $(p<0.05)$ after $48 \mathrm{~h}$ of induction of wound. The EE shows to be active as $\mathrm{DPPH}$ radical scavenger. The $\mathrm{IC}_{50}$ value for the $\mathrm{EE}$ was similar to the $\mathrm{IC}_{50}$ value of gallic acid (1.04 \pm 0.27 and $1.15 \pm 0.02 \mu \mathrm{g} \mathrm{mL}^{-1} \mathrm{DPPH}$, respectively). In conclusion, the EE of Sideroxylon obtusifolium shows local anti-inflammatory activity in the model of wound healing in rats, in addition to antioxidant potential.

Key words: Cell migration, inflammation, myeloperoxidase, Sapotaceae, Sideroxylon, wound healing. 


\section{INTRODUÇÃO}

A cicatrização, processo de reparo que segue a lesão na pele e em outros tecidos moles (Dash \& Murthy, 2011), é um processo biológico complexo dividido em fases que se sobrepõem: hemostasia e inflamação controlada, formação de tecido novo e remodelamento. A primeira fase envolve interações entre diferentes tipos celulares e matriz extracelular com liberação de mediadores inflamatórios. A segunda fase inclui reepitelização, formação de tecido de granulação e matriz extracelular, além de angiogênese. E finalmente, na terceira fase, ocorre substituição do colágeno, apoptose e formação da cicatriz (Gurtner et al., 2008; Behm et al., 2011; Ebeling et al., 2014).

Adicionalmente aos remédios convencionais, os fitoterápicos podem ser uma alternativa interessante ou co-adjuvante benéfica em diferentes fases da cicatrização de feridas. Mais de $80 \%$ da população mundial continua a depender de medicamentos tradicionais para várias doenças de pele (Shamuga Priya et al., 2002).

A espécie Sideroxylon obtusifolium (Roemer \& Schultes) T.D. Penn. (Sapotaceae), popularmente conhecida como "quixabeira", "quixaba" ou "rompegibão", é encontrada na Caatinga do São Francisco e de todo o Nordeste, nas restingas litorâneas e no pantanal Mato-grossense, entre outras regiões. A entrecasca desta espécie é usada na medicina popular no interior do Nordeste, pelas populações menos favorecidas, principalmente no tratamento de inflamações ovarianas, diabetes, gripe, gastrite, dor nos rins e pancadas (Braga, 1976; Mors et al., 2000; Albuquerque \& Oliveira, 2007).

Em estudo anterior nós demonstramos que o extrato etanólico da entrecasca da $S$. obtusifolium apresenta atividades antinociceptica e anti-inflamatória, após tratamento oral único em roedores, suportando o uso popular desta espécie para tratar desordens inflamatórias (Araújo-Neto et al., 2010).

Com base nas considerações acima, decidimos investigar a habilidade cicatrizante e anti-inflamatória local do extrato etanólico (EE) da entrecasca da $S$. obtusifolium após administração tópica sobre lesões de pele em ratos.

\section{MATERIAL E MÉTODO}

Coleta e identificação do material vegetal A entrecasca da planta Sideroxylon obtusifolium (Roemer \& Schultes) T.D. Penn. (Sapotaceae), conhecida como "quixabeira", foi coletada em março de 2009, no Município de Canindé de São Francisco, Estado de Sergipe (0966'00"S, 3778'94'O). Um espécime foi registrado no Herbário da Universidade Federal de Sergipe, com o número ASE 13.163, e a identificação botânica realizada pela botânica Dre Ana Paula do Nascimento Prata, do Departamento de Biologia da Universidade Federal de Sergipe.

\section{Preparação do extrato etanólico}

As amostras de entrecasca foram submetidas à secagem em estufa a $37^{\circ} \mathrm{C}$, com renovação e circulação de ar até completa desidratação. A entrecasca seca $(2,8 \mathrm{~kg})$ foi reduzida a pó utilizando-se um moinho de facas e, posteriormente, submetida à extração com etanol a $90 \%$ durante 5 dias, por maceração exaustiva. Após este período, o material foi filtrado e concentrado em rota-evaporador a $50^{\circ} \mathrm{C}$ sob pressão reduzida, obtendo-se 459,6 g (16,4\%) do extrato etanólico.

\section{Prospecção fitoquímica}

O extrato da planta utilizada neste trabalho foi submetido à prospecção fitoquímica, seguindo a descrição de Matos (1997), com objetivo de detectar a ocorrência de diversos constituintes químicos presentes no EE da entrecasca de S. obtusifolium.

\section{Animais experimentais}

Os ratos Wistar (180-300 g), adultos, machos, foram obtidos do Biotério Central da Universidade de São Paulo (USP, São Paulo, Brasil) e levados ao Biotério local do Departamento de Farmacologia da USP, onde permaneceram por pelo menos 2 dias antes da realização dos experimentos. Os animais foram aleatoriamente divididos em grupos, mantidos em caixas plásticas (5 ratos/caixa) em sala com temperatura controlada $\left(21 \pm 2^{\circ} \mathrm{C}\right)$, sob ciclo natural claro/escuro $12 / 12 \mathrm{~h}$, troca diária de serragem e água e ração ad libitum. Os procedimentos experimentais foram conduzidos de acordo com as normas de procedimentos sobre cuidados com os animais para uso de animal em pesquisa. Todos os esforços foram realizados para minimizar o sofrimento dos animais.

Para o desenvolvimento deste projeto, o mesmo foi submetido e aprovado pelo Comitê de Ética em Pesquisa com Animais (CEPA) da Universidade Federal de Sergipe sob o número de protocolo 47/07.

\section{Ferida e cicatrização cutânea}

O modelo de ferida e cicatrização cutânea empregado foi descrito previamente por Muscará et al. (2000). Após os animais terem sido anestesiados com xilazina e cetamina pela via intraperitoneal (16 $\mathrm{mg} \mathrm{kg}^{-1}$ e $80 \mathrm{mg} \mathrm{kg}^{-1}$, respectivamente), foi realizada uma incisão na pele dorsal de $6 \mathrm{~cm}$. A seguir, foram inseridos subcutaneamente discos de esponja de 
álcool polivinílico (10 $\mathrm{mm}$ de diâmetro por $4-5 \mathrm{~mm}$ de espessura, previamente auto-clavados por 20 min) embebidos em solução salina, os quais foram alinhados longitudinalmente em ambos os lados da ferida, perfazendo um total de 8 discos. Em seguida, as feridas foram suturadas com 10 pontos cirúrgicos e os animais ( $n=6-10 / g r u p o$ ) submetidos aos diferentes tratamentos que consistiram na aplicação tópica, 2 vezes ao dia, de $0,4 \mathrm{~mL}$ de creme Lanette ${ }^{\circledR}$ (veículo), EE de S. obtusifolium

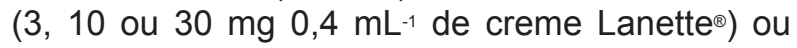
pomada comercial Novaderm ${ }^{\circledast}$ (acetato de clostebol e sulfato de neomicina, ambos $5 \mathrm{mg} \mathrm{g}^{-1}$ ). Após $48 \mathrm{~h}$ da indução da ferida, os animais foram novamente anestesiados com xilazina e cetamina $\left(16 \mathrm{mg} \mathrm{kg}^{-1}\right.$ e $80 \mathrm{mg} \mathrm{kg}^{-1}$, respectivamente), eutanasiados, e a pele dorsal removida cuidadosamente, bem como as esponjas para coleta do exsudato.

\section{Preparo dos cremes contendo o extrato}

O EE da S. obtusifolium (3, 10 e $30 \mathrm{mg}$ ) foi incorporado à $0,4 \mathrm{~mL}$ de creme Lanette ${ }^{\circledR}$ (creme hidratante constituído de base aniônica para incorporar ativos) em graal de porcelana até se obter preparações homogêneas para serem aplicadas topicamente nos animais.

\section{Medida de tensão}

O grau de cicatrização foi avaliado medindose a tensão necessária aplicada para a ruptura (reabertura) da ferida. Para isso, foi adaptado um analgesímetro eletrônico (Insight, Ribeirão Preto, $\mathrm{SP})$ que possui um transdutor de força conectado a um painel digital. A pele dos ratos no local da ferida foi cortada em tiras de $1 \mathrm{~cm}$, e estas foram alocadas em um dispositivo desenvolvido no laboratório para a medida de tensão. Neste dispositivo, os extremos da pele ficaram presos a dois binder clips revestidos com tela esmeril (Norton K"246, $n^{\circ} 36$ ), sendo ambos presos por hastes de metal. Uma das bases ficou parafusada a uma base de madeira e a outra ficou livre de atrito para ser movimentada. A ponteira do analgesímetro foi apoiada na base livre e a tensão foi gradualmente aumentada até as partes do tecido cederem, sendo o valor da força máxima aplicada registrado pelo analgesímetro. Os valores de tensão estão expressos no valor equivalente em gramas que foi aplicado a base móvel.

\section{(MPO)}

Medida da atividade de mieloperoxidase

As esponjas que foram retiradas dos animais foram espremidas com pinças para obtenção do exsudato (aproximadamente $2 \mathrm{~mL}$ de exsudato/animal), o qual foi utilizado na avaliação da atividade de MPO. À $50 \mu \mathrm{L}$ de exsudato foi adicionado igual volume de tampão fosfato de potássio $(50 \mathrm{mM}, \mathrm{pH} 6,0)$ contendo $0,5 \%$ de brometo de hexadeciltrimetilamônio (HTAB; Sigma Chem. Co., EUA). A seguir, as amostras foram aquecidas durante $2 \mathrm{~h}$ a $60^{\circ} \mathrm{C}$ em estufa (para inativação de catalase endógena) e centrifugados a $10.000 x g$ durante $5 \mathrm{~min}$. Após a centrifugação, os sobrenadantes foram coletados e usados como fonte para medida da atividade de MPO. Dez microlitros desta solução foram adicionados a $200 \mu \mathrm{L}$ de tampão fosfato de potássio, $\mathrm{pH} 6,0$, contendo 0,164 $\mathrm{mg} \mathrm{mL}^{-1}$ de di-hidrocloreto de o-dianisidina (Sigma Chemical Co., EUA) e 0,0005\% de água oxigenada (Merck, Alemanha). A mudança de absorbância (DO) foi medida em um leitor de placa (Espectra max plus 384, EUA) a $460 \mathrm{~nm}$ durante $5 \mathrm{~min}$ a intervalos de $10 \mathrm{~s}$. Os resultados estão expressos como unidades (U) de MPO por $\mathrm{mL}$ de exsudato. Uma unidade de MPO foi definida como aquela capaz de degradar 1 $\mu \mathrm{mol}$ de $\mathrm{H}_{2} \mathrm{O}_{2}$ por min a $25^{\circ} \mathrm{C}$ (Bradley et al.,1982).

\section{Contagem de leucócitos}

As contagens total e diferencial de leucócitos foram realizadas nos exsudatos das esponjas. A contagem total de leucócitos foi realizada em câmara de Neubauer e a contagem diferencial foi realizada em esfregaços preparados em citocentrífuga (800 rpm) e corados com corante comercial Panótico ${ }^{\circledR}$. Os esfregaços foram submetidos a leitura em microscópio óptico, baseada na morfologia normal dos leucócitos, de pelo menos 100 células. Os resultados estão expressos como número de células por $\mathrm{mL}$ de exsudato.

\section{Atividade antioxidante}

A análise quantitativa da atividade antioxidante foi baseada no método descrito por Brand-Williams et al. (1995), com pequenas modificações. O sequestro do radical 2,2-difenil-1picrilhidrazil (DPPH) foi seguido pelo monitoramento da diminuição na absorbância a $515 \mathrm{~nm}$, o que ocorre devido à redução pelo antioxidante.

A curva de calibração foi estabelecida através da preparação de diluições de uma solução estoque do radical DPPH $\left(40 \mu \mathrm{g} \mathrm{mL}^{-1}\right)$ para obter concentrações finais de $5,10,15,20,25$ e 30 $\mu \mathrm{g} \mathrm{mL} \mathrm{m}^{-1}$. A absorbância de cada concentração foi então monitorada em um espectrofotômetro (UV BEL Photonics 1105) a $515 \mathrm{~nm}$. As medidas foram realizadas em triplicata com intervalos de $1 \mathrm{~min}$. A equação da concentração $\times$ absorbância da curva de calibração para o radical DPPH foi $C=110,547$ $-0,02804 \times A$, onde $C$ é a concentração do radical DPPH no meio e A é a absorbância a $515 \mathrm{~nm}$. O coeficiente de correlação foi de $R=0,9983$.

O EE da entrecasca da $S$. obtusifolium foi diluído em metanol para obtermos concentrações de $5,10,15,20,25$ e $30 \mu \mathrm{g} \mathrm{mL}^{-1}$. O desaparecimento

Rev. Bras. PI. Med., Campinas, v.17, n.1, p.164-170, 2015. 
do radical DPPH foi monitorado pela diminuição da absorção a $515 \mathrm{~nm}$, o qual foi registado após 0,1 , 5 e $10 \mathrm{~min}$ e, posteriormente, a cada $10 \mathrm{~min}$ até 1 h. O controle negativo foi metanol puro utilizado no preparo das amostras, enquanto o controle positivo foi o ácido gálico em metanol nas concentrações de $5,10,15,20,25$ e $30 \mu \mathrm{g} \mathrm{mL}^{-1}$. A mistura de metanol e EE foi usada como branco.

A concentração do radical DPPH na mistura reacional foi calculada com base na curva de calibração, onde a [DPPH] é expressa em $\mu \mathrm{g}$ $\mathrm{mL}^{-1}$. A porcentagem de DPPH remanescente $(\%$ $\mathrm{DPPH}_{\mathrm{REM}}$ ) foi calculada de acordo com BrandWilliams et al. (1995), como se segue: \% $\mathrm{DPPH}_{\mathrm{REM}}$ $=[\mathrm{DPPH}]_{\mathrm{T}} /[\mathrm{DPPH}]_{\mathrm{T}} \times 100$, onde $\mathrm{T}$ é o tempo no qual a absorbância foi determinada (1-60 min) e T0 é o tempo zero. A quantidade de antioxidante necessária para diminuir a concentração inicial do radical DPPH para $50 \%\left(\mathrm{IC}_{50}\right)$ foi calculada utilizando a \% $\mathrm{DPPH}_{\text {RеM }}$ no tempo de $60 \mathrm{~min}$. Os resultados estão expressos como $\mu \mathrm{g}$ de antioxidante $\mathrm{mL}^{-1}$ de $\mathrm{DPPH} \pm$ desvio padrão.

\section{Análise estatística}

Os resultados das atividades cicatrizante e anti-inflamatória tópica estão expressos como média \pm E.P.M. de $n$ animais por grupo. A atividade antioxidante foi realizada utilizando a versão 7.5 Origem (Microcal, Northampton, MA, EUA) e os valores estão apresentados como a média \pm desvio padrão (triplicata). A avaliação estatística dos dados foi realizada usando a análise de variância (ANOVA) para múltiplas comparações, seguida do teste de Bonferroni. Valores de $p$ menores que 0,05 foram considerados significativos.

\section{RESULTADO E DISCUSSÃO}

Nós demonstramos pela primeira vez neste estudo que o EE da S. obtusifolium apresenta atividade anti-inflamatória sobre lesões de pele em ratos, diminuindo o infiltrado leucocitário, após administração tópica. Adicionalmente, mostramos que este extrato apresenta atividade antioxidante.

A S. obtusifolium apresenta importância etnofarmacológica atribuída a seus metabólitos secundários (Albuquerque et al., 2007). Os compostos ativos presentes na espécie $S$. obtusifolium mostram-se efetivos em processos inflamatórios e dolorosos (Araújo-Neto et al., 2010). Neste estudo, a análise fitoquímica do EE bruto da $S$. obtusifolium mostrou presença de fenóis totais, taninos, flavonóis, flavanonóis, flavanonas, xantonas, esteroides, triterpenóides e heterosídeos saponínicos.

A aplicação tópica de 3,10 ou $30 \mathrm{mg} \mathrm{0,4}$ $\mathrm{mL}^{-1}$ de creme Lanette ${ }^{\circledR}$ do EE da entrecasca da S. obtusifolium, 2 vezes ao dia, não apresentou atividade cicatrizante significativa após $48 \mathrm{~h}$ da indução da ferida em ratos, ou seja, não foi capaz de aumentar a tensão de ruptura das cicatrizes na pele dorsal de ratos (Figura 1). Diferentemente, a preparação comercial Novaderm ${ }^{\circledast}$ foi capaz de

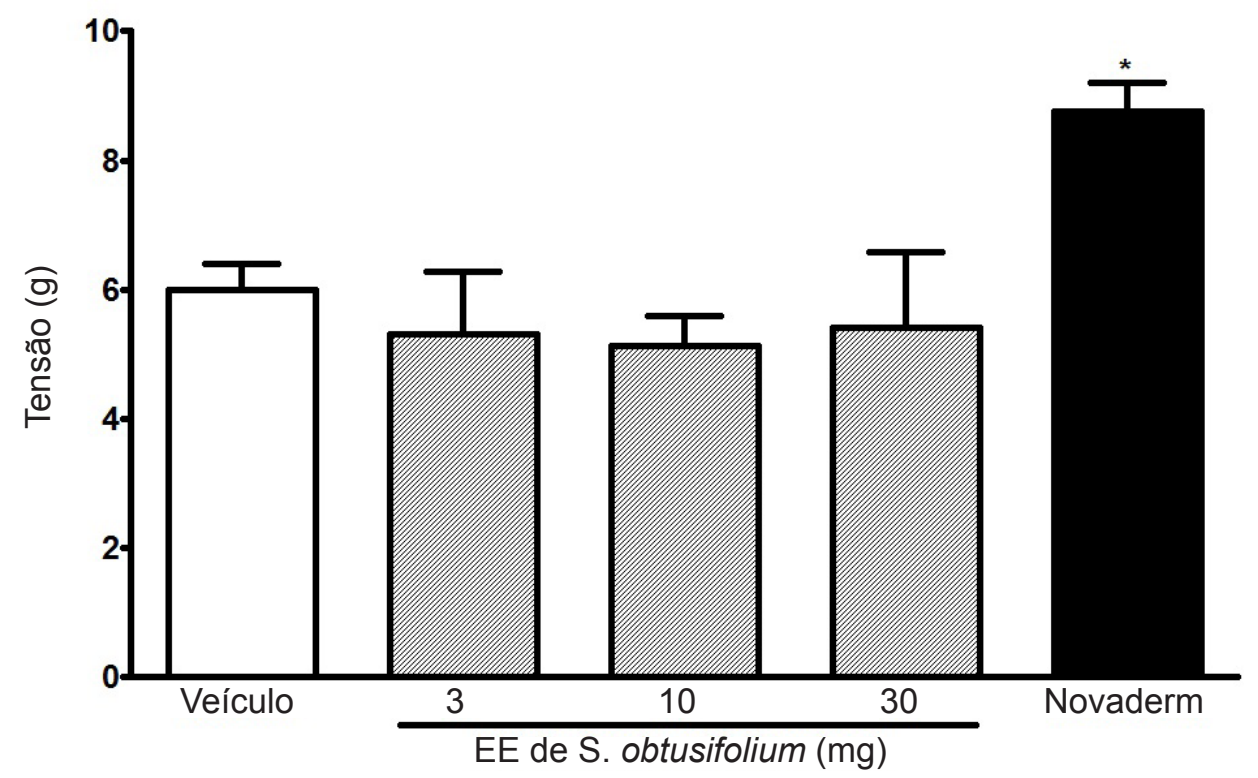

FIGURA 1. O efeito do extrato etanólico (EE) da Sideroxylon obtusifolium sobre a tensão de ruptura das cicatrizes na pele dorsal de ratos após $48 \mathrm{~h}$ de indução da ferida. Os ratos foram tratados, 2 vezes ao dia, com veículo

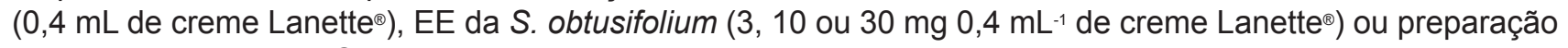
comercial Novaderm ${ }^{\circledast}$. Os resultados estão expressos como média \pm EPM da tensão de ruptura em g. ${ }^{*} p<0,05$ comparado ao grupo veículo ( $n=6-10 / g r u p o)$.

Rev. Bras. PI. Med., Campinas, v.17, n.1, p.164-170, 2015. 
aumentar $(p<0,05)$ a tensão necessária para romper a cicatriz, quando comparado ao grupo de animais que recebeu apenas o veículo (grupo controle) (Figura 1). Estudo preliminar do nosso grupo mostra que os valores de tensão na pele de animais controle aumentam para cerca de $40 \mathrm{~g}$ com a preparação Novaderm ${ }^{\oplus}, 5$ dias após a indução das feridas.

Estudo anterior demonstra que o extrato metanólico da Mimusops elengi Linn., uma outra espécie da família Sapotaceae, é eficaz na cicatrização de feridas induzidas por incisão, após 10 dias de tratamento diário (Gupta \& Jain, 2011). Sendo assim, podemos inferir que talvez em tempos superiores de análise, o EE da entrecasca da S. obtusifolium pudesse induzir algum efeito cicatrizante mediante a administração tópica.

O processo flogístico foi evidente em todos os animais submetidos à indução de feridas após $48 \mathrm{~h}$, com edema e vermelhidão que caracterizam a inflamação. As fases inflamatória, proliferativa e de maturação da cura são dependes do tipo e da extensão do dano, do estado de saúde do indivíduo e do potencial do tecido em reparar (Swamya et al., 2007). A intervenção em qualquer uma destas fases por drogas poderia, eventualmente, levar a uma promoção no processo de cura (Mukherjee et al., 2000). Portanto, é importante considerar o processo inflamatório envolvido na formação de cicatriz.

No modelo de ferida e cicatrização utilizado, a migração de células para a região da ferida foi avaliada através da inserção de discos de esponjas de álcool polivinílico, conforme utilizado previamente por Muscará et al. (2000).

Neste estudo, o tratamento tópico dos

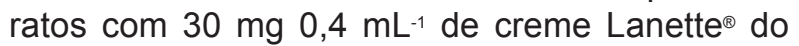
EE da S. obtusifolium, 2 vezes ao dia, diminuiu de forma significativa $(p<0,01)$ a atividade de MPO, um indicador de neutrófilos, após $48 \mathrm{~h}$ da indução da ferida em ratos (Figura 2). A preparação comercial Novaderm ${ }^{\circledast}$ também foi capaz de diminuir $(p<0,01)$ a atividade de MPO, quando comparado ao grupo de animais controle (veículo) (Figura 2).

Para confirmar o papel do EE da $S$. obtusifolium na migração de leucócitos, contagens total e diferencial de leucócitos presentes no exsudato retirado das esponjas foram realizadas. Observamos que com a aplicação tópica de $30 \mathrm{mg}$ $0,4 \mathrm{~mL}^{-1}$ de creme Lanette ${ }^{\circledR}$ do EE da S. obtusifolium, 2 vezes ao dia, igualmente ao observado na avaliação da atividade de MPO, houve diminuição significativa no número de leucócitos totais $(p<0,05)$ e de células polimorfonucleares $(p<0,01)$ (Figura 3 ). O grupo que recebeu aplicação tópica da preparação Novaderm ${ }^{\circledast}$, quando comparado ao grupo de animais que recebeu apenas veículo, também apresentou diminuição de leucócitos totais $(p<0,01)$ e de polimorfonucleares $(p<0,001)$ (Figura 3$)$. Portanto, o EE da S. obtusifolium foi eficaz na diminuição do processo inflamatório como é evidente pela notável redução do infiltrado de leucócitos. Estes dados estão de acordo com resultados anteriores do nosso grupo de pesquisa, onde evidenciamos propriedades anti-inflamatórias produzidas pelo EE da entrecasca da S. obtusifolium, como diminuição

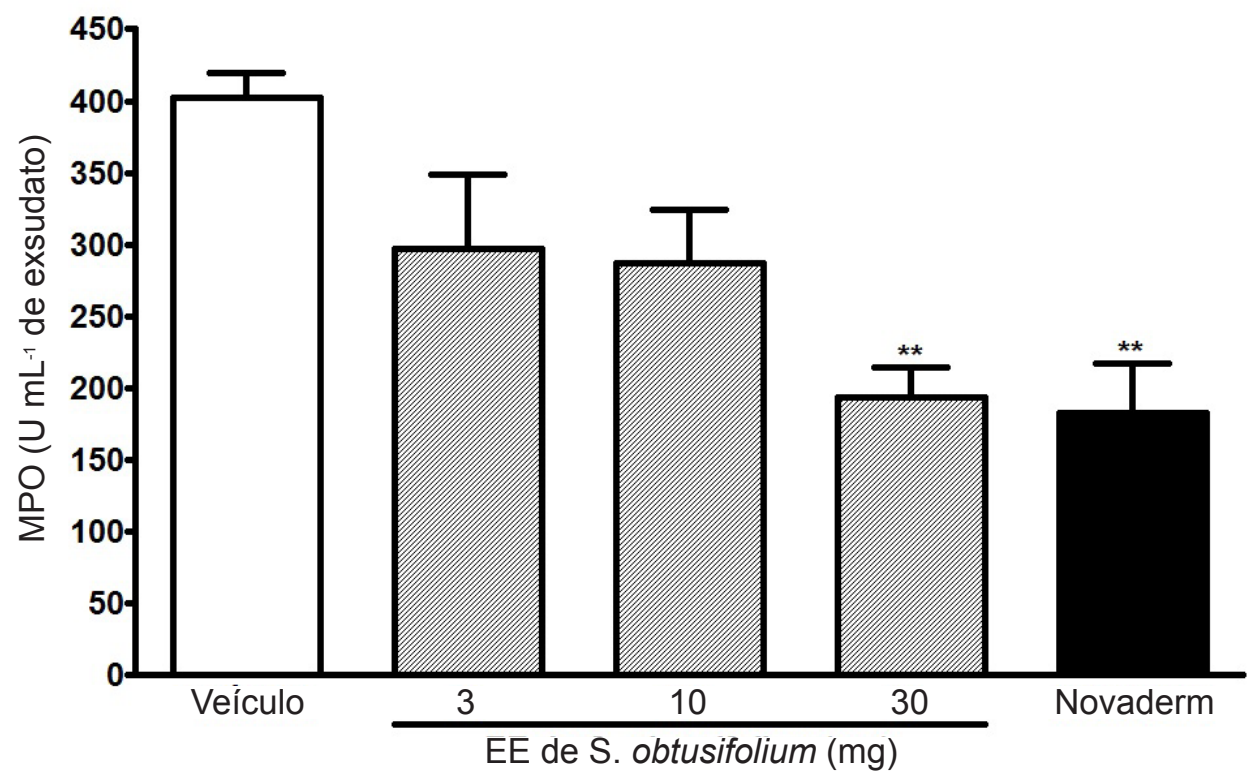

FIGURA 2. O efeito do extrato etanólico (EE) da Sideroxylon obtusifolium sobre a atividade da mieloperoxidase (MPO) após $48 \mathrm{~h}$ de indução da ferida. Os ratos foram tratados, 2 vezes ao dia, com veículo $(0,4 \mathrm{~mL}$ de creme

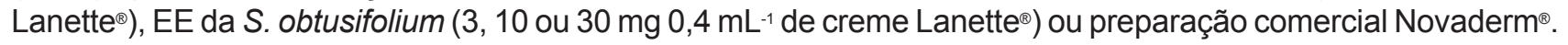
Os resultados estão expressos como média \pm EPM da atividade de MPO em unidades $(U) \mathrm{mL}^{-1}$ de exsudato. ${ }^{* *} p<0,01$ comparado ao grupo veículo ( $n=6-10 / g r u p o$ ). 
do infiltrado leucocitário peritoneal e edema de pata induzidos por carragenina (Araújo-Neto et al., 2010), e diminuição da atividade de MPO na bexiga urinária de animais com cistite hemorrágica induzida por ciclofosfamida (Pereira et al., 2013), quando administrado por via oral em ratos. Essas ações anti-inflamatórias do EE se devem, ao menos em parte, aos efeitos de seus constituintes, taninos e flavonoides.

Neste estudo, quando avaliamos a capacidade antioxidante do EE da S. obtusifolium, observamos que $\mathrm{O}$ valor de $\mathrm{IC}_{50}$ foi similar ao do composto de referência ácido gálico $(1,04 \pm 0,27$ e $1,15 \pm 0,02 \mu \mathrm{g} \mathrm{mL}^{-1}$ de DPPH, respectivamente, em triplicata). O EE apresentou cinética comportamental rápida, atingindo seu estado de equilíbrio em 10 min (Tabela 1). Concordando com este resultado, Desmarchelier et al. (1999) demonstraram que a S. obtusifolium é efetiva no sequestro de radical livre, como inibidora de danos ao DNA e da peroxidação lipídica, sugerindo que estas atividades antioxidantes possam desempenhar um papel importante na atividade anti-inflamatória desta espécie.

O estudo fitoquímico do EE da entrecasca da $S$. obtusifolium detectou a presença de flavonoides, os quais são relatados como bons antioxidantes e anti-inflamatórios (Nijveldt et al., 2001; Odontuya et al., 2005; Soobrattee et al., 2005). Os flavonoides inibem a ciclo-oxigenase (COX)-2, óxido nítrico sintase induzível (iNOS), lipoxigenase, mono-oxigenase microssomal, glutationa S-transferase, succinoxidase mitocondrial e NADPH-oxidase (Odontuya et al., 2005; Soobrattee et al., 2005). Além disso, os flavonoides possuem capacidade de inibir a desgranulação de neutrófilos e reduzir a ativação do complemento, diminuindo assim a adesão de células inflamatórias ao endotélio e a resposta inflamatória (Nijveldt et al., 2001). Os flavonoides também inibem a produção de metaloproteinase- 9 em queratinócitos humano durante o processo de cicatrização de feridas (Majtan et al., 2013). Taninos, também presentes no EE da S. obtusifolium, são capazes de inibir as enzimas COX-2 e/ou iNOS (Carvalho, 2004). Aos taninos e saponinas presentes em extratos obtidos da casca da Mimosa tenuiflora (Willd.) Poiret (Leguminoseae) também são atribuídas propriedades cicatrizantes (Rivera-Arce et al., 2007).

\section{CONCLUSÃO}

Em conclusão, os resultados do presente estudo mostram pela primeira vez que o EE da entrecasca da S. obtusifolium atua na fase inflamatória do processo de cicatrização de feridas, interferindo na migração celular, após administração tópica em ratos, com potencial de cura de feridas. Entretanto são necessários estudos futuros para elucidar o mecanismo de ação anti-inflamatório desta espécie na cura de feridas.

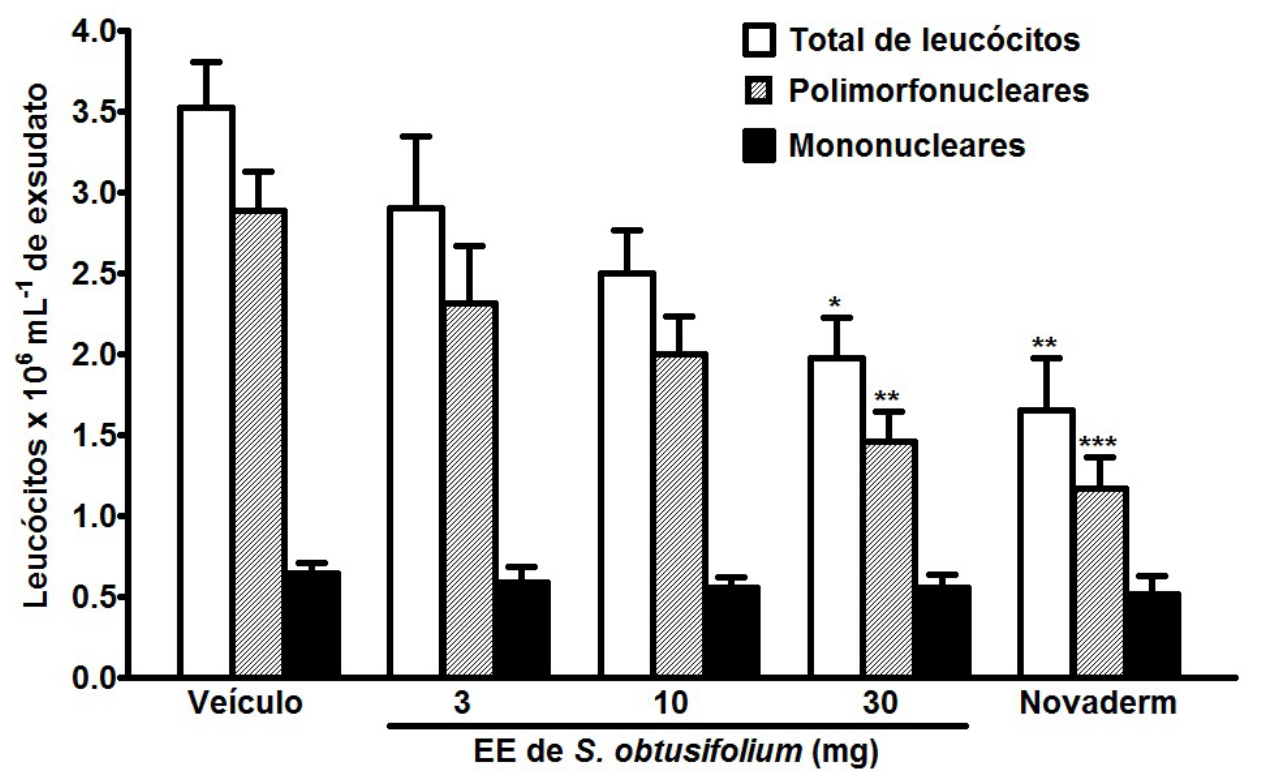

FIGURA 3. O efeito do extrato etanólico (EE) da Sideroxylon obtusifolium sobre o número de leucócitos após $48 \mathrm{~h}$ de indução da ferida. Os ratos foram tratados, 2 vezes ao dia, com veículo $\left(0,4 \mathrm{~mL}\right.$ de creme Lanette $\left.{ }^{\circledR}\right)$,

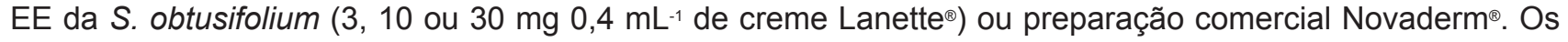
resultados estão expressos como média \pm EPM do número $\left(\times 10^{6}\right)$ de leucócitos totais, células polimorfonucleares e células mononucleares $\mathrm{mL}^{-1}$ de exsudato. ${ }^{*} \mathrm{p}<0,05,{ }^{* *} \mathrm{p}<0,01 \mathrm{e}^{* * *} \mathrm{p}<0,001$ comparado ao respectivo grupo veículo ( $n=6-10 /$ grupo). 
TABELA 1. Cinética comportamental do extrato etanólico (EE, $25 \mathrm{gg} \mathrm{mL}^{-1}$ ) da S. obtusifolium e ácido gálico $\left(25 \mu \mathrm{g} \mathrm{mL}^{-1}\right)$ frente ao radical livre DPPH.

\begin{tabular}{lcc}
\hline \multirow{2}{*}{ Tempo $(\mathbf{m i n})$} & \multicolumn{2}{c}{$\% \mathrm{DPPH}_{\mathrm{REM}}$ (média \pm desvio padrão) } \\
\cline { 2 - 3 } & EE & Ácido Gálico \\
\hline 1 & $23,09 \pm 5,32$ & $11,71 \pm 0,10$ \\
5 & $15,90 \pm 2,71$ & $10,81 \pm 0,11$ \\
10 & $12,04 \pm 1,33$ & $9,76 \pm 0,05$ \\
20 & $10,64 \pm 0,88$ & $7,61 \pm 0,04$ \\
30 & $10,02 \pm 0,68$ & $6,04 \pm 0,03$ \\
40 & $9,73 \pm 0,66$ & $5,76 \pm 0,04$ \\
50 & $9,32 \pm 0,59$ & $5,63 \pm 0,03$ \\
60 & $9,39 \pm 0,60$ & $5,37 \pm 0,01$ \\
\hline
\end{tabular}

\section{AGRADECIMENTO}

Os autores agradecem à Coordenação de Aperfeiçoamento de Pessoal de Nível Superior - CAPES (Ações Novas Fronteiras - Programa de Cooperação Acadêmica - PROCAD/NF 2007), ao Conselho Nacional de Desenvolvimento Científico e Tecnológico - CNPq (Edital MCT/CNPq 15/2007 - Universal) e à Universidade Federal de Sergipe (Edital PAIRD 2007) pela concessão de auxílios financeiros para execução deste trabalho. SKPC, MNM e SMT são beneficiários de bolsa de produtividade do CNPq.

\section{REFERÊNCIAS}

ALBUQUERQUE, U.P.; OLIVEIRA, R.F. Is the use-impact on native caatinga species in Brazil reduced by the high species richness of medicinal plants? Journal of ethnopharmacology, v.113, n.1, p.156-70, 2007.

ALBUQUERQUE, U.P. et al. Medicinal plants of the caatinga (semi-arid) vegetation of NE Brazil: A quantitative approach. Journal of ethnopharmacology, v.114, p.325-54, 2007.

ARAUJO-NETO, V. et al. Therapeutic benefits of Sideroxylon obtusifolium (Humb. ex Roem. \& Schult.) T.D. Penn., Sapotaceae, in experimental models of pain and inflammation. Revista Brasileira de Farmacognosia, v.20, p.933-8, 2010.

BEHM, B. et al. Cytokines, chemokines and growth factors in wound healing. Journal of the European Academy of Dermatology and Venereology, v.26, p.812-20, 2011.

BRADLEY, P.P. et al. Measurement of cutaneous inflammation: estimation of neutrophil content with an enzyme marker. The Journal of Investigative Dermatology, v.78, n.3, p.206-9, 1982.

BRAGA, R.A. Plantas do Nordeste, especialmente do Ceará. 3.ed. Mossoró: Coleção Mossoroense, 1976. $540 \mathrm{p}$.

BRAND-WILLIAMS, W. et al. Use of a free radical method to evaluate antioxidant activity. Lebensmittel Wissenschaft Technologie, v.28, p.25-30, 1995.
CARVALHO, J.C.T. Fitoterápicos anti-inflamatórios: aspectos químicos, farmacológicos e aplicações terapêuticas. Ribeirão Preto: Tecmedd Editora, 2004. 480p.

DASH, G.K.; MURTHY, P.N. Studies on Wound Healing Activity of Heliotropium indicum Linn. Leaves on Rats. International Scholarly Research Network Pharmacology, Article ID 847980, p.1-8, 2011.

DESMARCHELIER, C. et al. Antioxidant and free radical scavenging activities in extracts from medicinal trees used in the 'Caatinga' region in northeastern Brazil. Journal of ethnopharmacology, v.67, p.69-77, 1999.

EBELING, S. et al. From a traditional medicinal plant to a rational drug: understanding the clinically proven wound healing efficacy of birch bark extract. PloS one, v.9, n.1, e86147, 2014

GUPTA, N.; JAIN, U.K. Investigation of wound healing activity of methanolic extract of stem bark of Mimusops elengi Linn. African journal of traditional, complementary, and alternative medicines, v.8, n.2, p.98-103, 2011.

GURTNER, G.C. et al. Wound repair and regeneration. Nature, v.453, p.314-21, 2008.

MAJTAN, J. et al. Fir honeydew honey flavonoids inhibit TNF-a-induced MMP-9 expression in human keratinocytes: a new action of honey in wound healing. Archives of dermatological research, v.305, n.7, p.619-27, 2013.

MATOS, F.J.A. Introdução à fitoquímica experimental. 2.ed. Fortaleza: UFC, 1997. 141p.

MORS, W.B. et al. Medicinal plants of Brazil. Michigan: Reference Publications, Inc., Algonac, 2000. 501p.

MUKHERJEE, P.K. et al. Evaluation of in-vivo wound healing activity of Hypericum patulum (Family: Hypericaceae) leaf extract on different wound model in rats. Journal of ethnopharmacology, v.70, p.315-21, 2000.

MUSCARÁ, M.N. et al. Wound collagen deposition in rats: effects of an NO-NSAID and a selective COX-2 inhibitor. British Journal of Pharmacology, v.129, p.681-6, 2000.

NIJVELDT, R.J. et al. Flavonoids: a review of probable mechanisms of action and potential applications. The American journal of clinical nutrition, v.74, p.41825, 2001.

ODONTUYA, G. et al. Structure-activity relationship for anti-inflammatory effect of luteolin and its derived glycosides. Phytotherapy research, v.19, p.782-6, 2005.

PEREIRA, D.S. et al. Effects of the ethanol extract of the inner bark of Syderoxylum obtusifolium in the cyclophosphamide-induced cystitis in rats. Journal of Medicinal Plant Research, v.7, p.1411-7, 2013.

RIVERA-ARCE, E. et al. Pharmacognostical studies of the plant drug Mimosae tenuiflorae cortex. Journal of ethnopharmacology, v.113, p.400-8, 2007.

SHAMUGA PRIYA, K. et al. Healing potential of Datura alba on burn wounds in albino rats. Journal of ethnopharmacology, v.83, p.193-9, 2002.

SOOBRATTEE, M.A. et al. Phenolics as potential antioxidant therapeutic agents: mechanism and actions. Mutation research, v.579, p.200-13, 2005.

SWAMYA, H.M.K. et al. Wound healing activity of embelin isolated from the ethanol extract of leaves of Embelia ribes burm. Journal of ethnopharmacology, v.109, p.529-34, 2007. 